\title{
Generating Functions of Lozenge Tilings for Hexagonal Regions via Nonintersecting Lattice Paths
}

\author{
Markus Fulmek \\ Fakultät für Mathematik, Oskar-Morgenstern-Platz 1, A-1090 Wien, Austria \\ Email: Markus.Fulmek@univie.ac.at \\ Received: January 19, 2021, Accepted: June 1, 2021, Published: June 18, 2021 \\ The authors: Released under the CC BY-ND license (International 4.0)
}

ABSTRACT: In a recent preprint, Lai showed that the quotient of generating functions of weighted lozenge tilings of two "half hexagons with lateral dents", which differ only in width, factors nicely, and the same is true for the quotient of generating functions of weighted lozenge tilings of two "quarter hexagons with lateral dents". Lai achieved this by using "graphical condensation".

The purpose of this paper is to exhibit how some of Lai's observations can be achieved by the LindströmGessel-Viennot method for nonintersecting lattice paths.

Keywords: Determinant evaluation; Generating functions; Lozenge tilings; Nonintersecting lattice paths 2020 Mathematics Subject Classification: 05A15

\section{Introduction}

In a recent preprint [4], Lai considers lozenge tilings of "half hexagons with lateral dents" and of "quarter hexagons with lateral dents" and shows that the quotient of the generating functions of such tilings (with a certain weight) for two objects (i.e., two "half" or two "quarter" hexagons) which differ only in width (to be explained below) has a nice factorization. We shall show how these observations can be obtained by the Lindström-Gessel-Viennot method [3,5] of nonintersecting lattice paths.

This paper is organized as follows:

- In section 2, we provide background information on lozenge tilings of hexagonal regions and nonintersecting lattice paths, present two of Lai's Theorems (regarding half hexagons and quarter hexagons, respectively), and show how the Lindström-Gessel-Viennot method applies to the case of half hexagons quite easily,

- in section 3, we work out the generating function corresponding to the case of quarter hexagons; this generating function involves a determinant whose evaluation is a bit involved,

- in section 4 , we state and prove an identity regarding the determinant appearing in the quarter hexagon case. This identity implies Lai's observation for quarter hexagons. Since the proof is quite long, it is subdivided in many subsections, which are meant to help in keeping track of the arguments.

\section{Exposition of Lai's results}

\subsection{Lozenge tilings of regions in the triangular lattice}

The literature on the enumerations of lozenge tilings is abundant (see, for instance, [1]). For the reader's convenience, we shall recall briefly the basic notions; see the left picture in Figure 1 and the left picture in Figure 2 for illustrations.

The triangular lattice is a tiling of the whole plane $\mathbb{R}^{2}$ with congruent equilateral triangles $\Delta_{i}$ of side length 1, i.e.,

$$
\mathbb{R}^{2}=\bigcup_{i \in \mathbb{N}} \Delta_{i} \text {, and for } i \neq j \text { the open interior of } \Delta_{i} \cap \Delta_{j} \text { is empty. }
$$


Without loss of generality, we may assume that all equilateral triangles have one side which is parallel to the horizontal axis (we adopt this assumption in all illustrations).

A region $R$ in this triangular lattice is the union of some subset of triangles.

A lozenge is the union $\Delta_{i} \cap \Delta_{j}$ of two different triangles that share an edge. Considering the angle between the horizontal axis and the shared edge of such lozenge, there are three possible orientations for lozenges:

- left-tilted (angle of shared edge $\frac{\pi}{3}$ ),

- right-tilted (angle of shared edge $\frac{2 \pi}{3}$ ),

- and vertical (angle of shared edge 0).

A tiling (in the sense presented above) of some region $R$ with lozenges is called a lozenge tiling of $R$. The basic task in this context is the enumeration (counting) of all lozenge tilings for some given region $R$. If we assign a weight $w(L)$ to all lozenges $L$ and define the weight $w(T)$ of some lozenge tiling $T$ to be the product of the weights of the lozenges it contains, i.e.,

$$
w(T):=\prod_{L \in T} w(L),
$$

then we can try to determine the generating function $\mathfrak{g} \mathfrak{f}(R, w)$ corresponding to the region $R$ and the weight $w$,

$$
\mathfrak{g} \mathfrak{f}(R, w):=\sum_{T} w(T)
$$

where the sum is over all lozenge tilings $T$ of $R$. (Enumeration can be viewed as the special case $w(L) \equiv 1$ for all lozenges $L$.)

Of particular interest are regions derived from hexagons, since the enumeration (or computation of the generating function) of their lozenge tilings turns out to give beautiful formulas in many cases.

\subsection{Lai's results}

\subsubsection{Hexagonal regions with dents}

In [4], Lai considered two regions derived from hexagons. These regions are

- the upper half (see the left picture in Figure 1. The hexagon is "cut in two pieces" along its horizontal symmetry axis)

- and the right half of the upper half (see the left picture in Figure 2. The upper half of the hexagon is "cut in two pieces" along its vertical symmetry axis)

of some given hexagon. We shall call such regions half hexagon and quarter hexagon, respectively.

Half hexagons and quarter hexagons can be tiled with lozenges if some triangles are removed.

- For half hexagons, remove triangles along with both non-horizontal sides (see the left picture in Figure 1),

- and for quarter hexagons, remove triangles along the right side (see the left picture in Figure 2).

In both cases, let us call such removed triangles dents, and the remaining regions dented half hexagon and dented quarter hexagon, respectively. Clearly, the positions of these dents can be encoded by a sequence of natural numbers.

Remark 2.1. The mere removal of triangles does not guarantee that the dented region has a lozenge tiling. The condition on the number and positions of dents that, in fact, guarantees the existence of lozenge tilings will be obvious "in the language of nonintersecting lattice paths", which we will describe in section 2.3.

Clearly, the generating function $\mathfrak{g} \mathfrak{f}(R, w)$ equals zero if the region $R$ has no lozenge tiling.

\subsubsection{Labels, weight functions and Theorems}

Assume that a cartesian coordinate system in the plane $\mathbb{R}^{2}$ is chosen such that the horizontal and vertical symmetry axis of the hexagon under consideration coincide with the horizontal and vertical axis, respectively, of the coordinate system. Then the vertical symmetry axis of a vertical lozenge $L$ intersects the horizontal axis of the coordinate system in a point $\left(\frac{k}{2}, 0\right)$, for some $k \in \mathbb{Z}$, we call this number $k$ the offset of the lozenge $L$ and denote it by $o(L)$.

For the "half hexagon", Lai assigned the weight $w_{h}$ 
- $w_{h}(L)=\frac{X q^{o(L)}+Y q^{-o(L)}}{2}$ to all vertical lozenges $L$,

- and $w_{h}(L)=1$ to all left- and right tilted lozenges $L$,

and established the following Theorem [4, Theorem 1.1], where the notation

$$
\mathfrak{P}_{q}(a, b, c):=\prod_{i=1}^{a} \prod_{j=1}^{b} \prod_{k=1}^{c} \frac{q^{i+j+k-1}-1}{q^{i+j+k-2}-1}
$$

is used (this formula is due to MacMahon [6], it gives the generating function of all plane partitions fitting in an $(a \times b \times c)$-box $)$ :

Theorem 2.1. For non-negative integers $x, m, n$ and two strictly increasing sequences $\mathbf{a}=\left(a_{i}\right)_{i=1}^{m}, \mathbf{b}=\left(b_{j}\right)_{j=1}^{n}$ of positive integers with $a_{m}, b_{n} \leq m+n$, we consider the half hexagon $S(\mathbf{a}, \mathbf{b}, x)$ with side lengths $x+m+$ $n, m+n, x, m+n$ and "dents" (missing triangles) in positions

- $a_{i}, i=1, \ldots m$ on the left side,

- $b_{j}, j=1, \ldots n$ on the right side.

(See the left picture in Figure 1, which illustrates the situation for $m=4, n=3, x=5, \mathbf{a}=(2,4,6,7)$ and $\mathbf{b}=(3,6,7)$.)

If the generating function

$$
\mathfrak{g} \mathfrak{f}\left(S(\mathbf{a}, \mathbf{b}, x), w_{h}\right):=\sum_{T} w_{h}(T)
$$

of all lozenge tilings $T$ of $S(\mathbf{a}, \mathbf{b}, x)$ is not zero, then we have for all non-negative integers $y$

$$
\begin{aligned}
\frac{\mathfrak{g f}\left(S(\mathbf{a}, \mathbf{b}, x), w_{h}\right)}{\mathfrak{g} f\left(S(\mathbf{a}, \mathbf{b}, y) w_{h}\right)} & =q^{(y-x)\left(\sum_{i=1}^{m} a_{i}+\sum_{j=1}^{n} b_{j}-(m+n)(m+n+1) / 2\right)} \frac{\mathfrak{P}_{q^{2}}(y, m, n)}{\mathfrak{P}_{q^{2}}(x, m, n)} \\
& \times \prod_{i=1}^{m} \frac{\left(q^{2 x+2 i} ; q^{2}\right)_{a_{i}-i}}{\left(q^{2 y+2 i} ; q^{2}\right)_{a_{i}-i}} \prod_{j=1}^{n} \frac{\left(q^{2 x+2 j} ; q^{2}\right)_{b_{j}-j}}{\left(q^{2 y+2 j} ; q^{2}\right)_{b_{j}-j}}
\end{aligned}
$$

Remark 2.2. Lai also presented another weight function for dented half hexagons, which led to Theorem 1.2 in [4]. While it is most likely that the nonintersecting lattice path approach also works for this other weight function, we shall not consider this Theorem here.

For the "quarter hexagon", Lai assigned the weight $w_{q}$

- $w_{q}(L)=\frac{q^{o(L)+1}+q^{-o(L)-1}}{2}$ to all vertical lozenges $L$,

- and $w_{q}(L)=1$ to all left- and right tilted lozenges $L$,

and established the following theorem [4, Theorem 1.3]:

Theorem 2.2. For non-negative integers $x, m$ and a strictly increasing sequence $\mathbf{a}=\left(a_{i}\right)_{i=1}^{m}$ of positive integers with $a_{m} \leq 2 m$, we consider the quarter hexagonal region $Q(\mathbf{a}, x)$ with side lengths $x+m, 2 m, x, 2 m$ and "dents" (missing triangles) in positions $a_{i}, i=1, \ldots m$ on the right side. (See the left picture in Figure 2 , which illustrates the situation for $x=4, m=6$ and $\mathbf{a}=(2,4,7,10,11,12)$.)

If the generating function

$$
\mathfrak{g} f\left(Q(\mathbf{a}, x), w_{q}\right):=\sum_{T} w(T)
$$

of all lozenge tilings $T$ of $Q(\mathbf{a}, x)$ is not zero, then we have for all non-negative integers $y$

$$
\frac{\mathfrak{g} \mathfrak{f}\left(Q(\mathbf{a}, x), w_{q}\right)}{\mathfrak{g} \mathfrak{f}\left(Q(\mathbf{b}, y), w_{q}\right)}=q^{2(y-x)\left(\sum_{i=1}^{m} a_{i}-m^{2}\right)} \prod_{i=1}^{m} \frac{\left(q^{4 y+2 a_{i}+2} ; q^{2}\right)_{2 i-a_{i}-1}}{\left(q^{4 x+2 a_{i}+2} ; q^{2}\right)_{2 i-a_{i}-1}}
$$

Remark 2.3. Also for dented quarter hexagons, Lai presented another weight function $w_{q}^{\prime}$ :

- $w_{q}^{\prime}(L)=\frac{q^{o(L)}+q^{-o(L)}}{2}$ to all vertical lozenges $L$ with $o(L) \neq 0$,

- $w_{q}^{\prime}(L)=\frac{1}{2}$ (not 1!) to all vertical lozenges $L$ with $o(L)=0$,

- and $w_{q}^{\prime}(L)=1$ to all left- and right tilted lozenges $L$, 

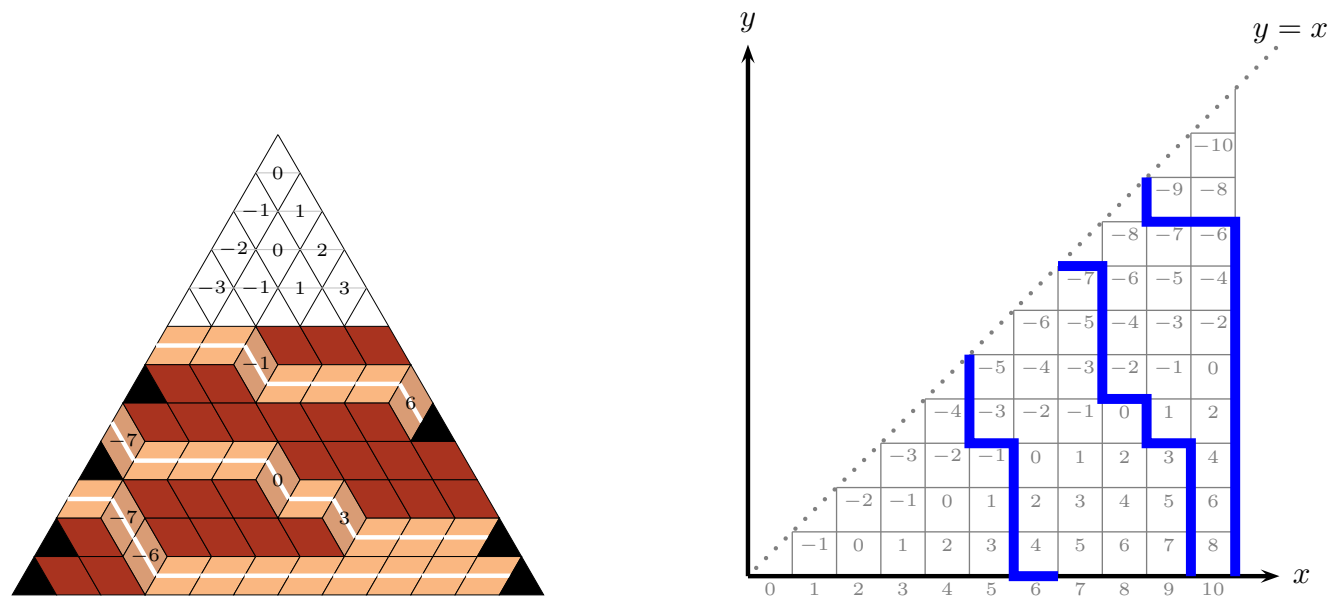

The pictures correspond to Figures 1.2.a and 2.1.a in Lai's preprint. The length of the upper horizontal side of the "half hexagon" in the left picture is the "width parameter" $x$ considered by Lai (so $x=5$ in this picture; the "height" by definition is equal to the length of the lateral sides, so it is 7 in this picture).

Figure 1: A lozenge tiling of a dented half hexagon in the triangular lattice, and the corresponding family of nonintersecting lattice paths.

which led to Theorem 1.4 in [4]. While it is clear that the nonintersecting lattice path approach also works for the modified weight function $w_{q}^{\prime}$, we shall not consider this Theorem here.

Lai provided proofs for the Theorems in [4] by "graphical condensation" (i.e., application of a certain Pfaffian identity to the enumeration of matchings). Lai's Theorems can be reformulated as follows. If only the width $x$ (i.e., the length of the upper horizontal side) of dented half or quarter hexagon is changed (i.e., the height and the positions of the lateral dents are unchanged), then the corresponding generating function changes by a simple product (which does not contain the variables $X$ or $Y$ occurring in $w_{h}$ ).

\subsection{Nonintersecting lattice paths, applied to the "half hexagon"}

While equation (1) looks quite complicated, it is rather easy to prove. We shall use its proof as an illustrating example for the nonintersecting lattice path approach.

\subsubsection{Translate lozenge tilings to nonintersecting lattice paths}

The literature on the connection between lozenge tilings and nonintersecting lattice paths is abundant (see, for instance, $[1$, Section 5]). Here, we shall demonstrate the weight-preserving bijection between lozenge tilings and nonintersecting lattice paths graphically (see Figure 1). The left picture in Figure 1 shows a half hexagon with side lengths $12,7,5,7$ in the triangular lattice. Its lateral sides have dents (i.e., missing triangles; indicated in the picture by black color), 4 on the left side in positions $(2,4,6,7)$ and 3 on the right side in positions $(3,6,7)$. (The large white triangle "on top" of this half hexagon is merely added to show the labeling of the vertical lozenges, which is constant vertically and increasing by 1 horizontally from left to right).

The picture also shows a lozenge tiling of this dented half hexagon, where the three possible orientations of lozenges (left-tilted, right-tilted and vertical) are indicated by three different colours. The lozenge tiling $T$ shown in the picture has weight

$$
w(T)=w_{-7}^{2} \cdot w_{-6} \cdot w_{-1} \cdot w_{0} \cdot w_{3} \cdot w_{6}
$$

where $w_{i}:=\frac{X q^{i}+Y q^{-i}}{2}$.

The lozenge tiling appears as a two-dimensional drawing of a surface in three-dimensional space, which consists of squares "glued together" along edges. In a three-dimensional coordinate system with axes $x, y$ and $z$, call the plane containing the $x$ - and $y$-axis the $x, y$-plane (and likewise for the other pairs of axes), and observe that

- left-tilted lozenges correspond to squares parallel to the $y, z$-plane,

- right-tilted lozenges correspond to squares parallel to the $x, y$-plane,

- and vertical lozenges correspond to squares parallel to the $x, z$-plane. 
Now observe that the tiling is uniquely determined by a collection of "ribbons"

- of squares parallel parallel to the $x, y$-plane (right-tilted lozenges)

- and of squares parallel parallel to the $x, z$-plane (vertical lozenges).

These "ribbons" are indicated by white lines for the tiling $T$ in the left picture of Figure 1; they correspond to lattice paths which do not have a point in common. Such lattice paths are called nonintersecting. The right picture in Figure 1 shows a "reflected, rotated and tilted" version of these paths in the lattice $\mathbb{Z} \times \mathbb{Z}$, where horizontal steps $(a, b) \rightarrow(a+1, b)$ are labelled $a-2 b$ (these labels are shown in the right picture only for the region of interest in our context, i.e., for $0 \leq y \leq x)$; vertical steps $(a, b) \rightarrow(a, b-1)$ are unlabeled.

It is easy to see that this correspondence (introduced here "graphically") between lozenge tilings and nonintersecting lattice paths in the lattice $\mathbb{Z} \times \mathbb{Z}$ (with horizontal steps $(a, b) \rightarrow(a+1, b)$ and vertical steps $(a, b) \rightarrow(a, b-1))$ is, in fact, a bijection, which is weight-preserving if we define the weight of some family $P$ of nonintersecting lattice paths as the product of $w_{i}$, where $i$ runs over the labels of all horizontal edges belonging to the paths in $P$.

\subsubsection{Derive the generating function of lattice paths recursively}

Clearly, the generating function $\mathfrak{g f}(a, b, c, d)$ of all lattice paths in the lattice $\mathbb{Z} \times \mathbb{Z}$ which start at point $(a, b)$ and end at point $(c, d)$ is zero for $a>c$ or $b<d$. For $a \leq c$ and $b \geq d$, it obeys the obvious recursion:

$$
\begin{aligned}
\mathfrak{g f}(a, b, a, d) & \equiv 1 \text { (only one lattice path, all steps vertical), } \\
\mathfrak{g} \mathfrak{f}(a, b, c, b) & =\prod_{i=a-2 b}^{c-2 b-1} \frac{X q^{i}+Y q^{-i}}{2}(\text { only one lattice path, all steps horizontal), } \\
\mathfrak{g f}(a, b, c, d) & =\frac{X q^{a-2 b}+Y q^{2 b-a}}{2} \mathfrak{g f}(a+1, b, c, d)+\mathfrak{g f}(a, b-1, c, d) .
\end{aligned}
$$

By straightforward verification we see that

$$
\mathfrak{g} \mathfrak{f}(a, b, c, d)=\prod_{j=1}^{c-a} \frac{\left(X q^{j-1-2 b+a}+Y q^{-j+1+2 d-a}\right)\left(1-q^{2(b-d)+2 j}\right)}{2\left(1-q^{2 j}\right)}
$$

obeys the above recursion.

\subsubsection{Specialize to paths corresponding to dented half hexagons}

We want to specialize (3) to the situation corresponding to dented half hexagons, i.e., to initial point $(a, a)$ and terminal point $(c, 0)$ (see the right picture in Figure 1): The generating function $\overline{\mathfrak{g} \mathfrak{f}}(a, c)$ of all lattice paths from $(a, a)$ to $(c, 0)$ is zero for $c<a$, and for $c \geq a$ it is equal to

$$
\overline{\mathfrak{g} f}(a, c)=\mathfrak{g f}(a, a, c, 0)=\left(2 q^{a}\right)^{(a-c)} \prod_{j=1}^{c-a} \frac{\left(X q^{j-1}+Y q^{1-j}\right)\left(1-q^{2 a+2 j}\right)}{1-q^{2 j}} .
$$

Note that increasing the width of the dented half hexagon by some $d \in \mathbb{N}$ corresponds bijectively to shifting all initial and terminal points of the corresponding nonintersecting lattice paths (i.e., $(a, a) \rightarrow(a+d, a+d)$ and $(c, 0) \rightarrow(c+d, 0))$, and from (4) we immediately obtain

$$
\overline{\mathfrak{g} \mathfrak{f}}(a+d, c+d)=\overline{\mathfrak{g} \mathfrak{f}}(a, c) \cdot \prod_{j=1}^{c-a} \frac{1-q^{2 a+2 d+2 j}}{q^{d}\left(1-q^{2 a+2 j}\right)} .
$$

\subsubsection{Rewrite the generating function with Pochhammer symbols}

By using the standard $q$-Pochhammer notation $(a ; q)_{0}:=1$ and

$$
\begin{aligned}
(a ; q)_{n} & :=\prod_{j=0}^{n-1}\left(1-a \cdot q^{j}\right) \\
(a ; q)_{-n} & :=\frac{1}{\left(a \cdot q^{-1} ; q^{-1}\right)_{n}}
\end{aligned}
$$

for integer $n>0$, we may rewrite (5) as follows:

$$
\overline{\mathfrak{g} \mathfrak{f}}(a+d, c+d)=\overline{\mathfrak{g} f}(a, c) \cdot \frac{\left(q^{2 d+2} ; q^{2}\right)_{c}}{q^{d c} \cdot\left(q^{2} ; q^{2}\right)_{c}} \cdot \frac{q^{d a} \cdot\left(q^{2} ; q^{2}\right)_{a}}{\left(q^{2 d+2} ; q^{2}\right)_{a}} .
$$

(Note that Lai uses a different notation. In [4, equation (1.1)], $q$ is replaced by $-q$.) 

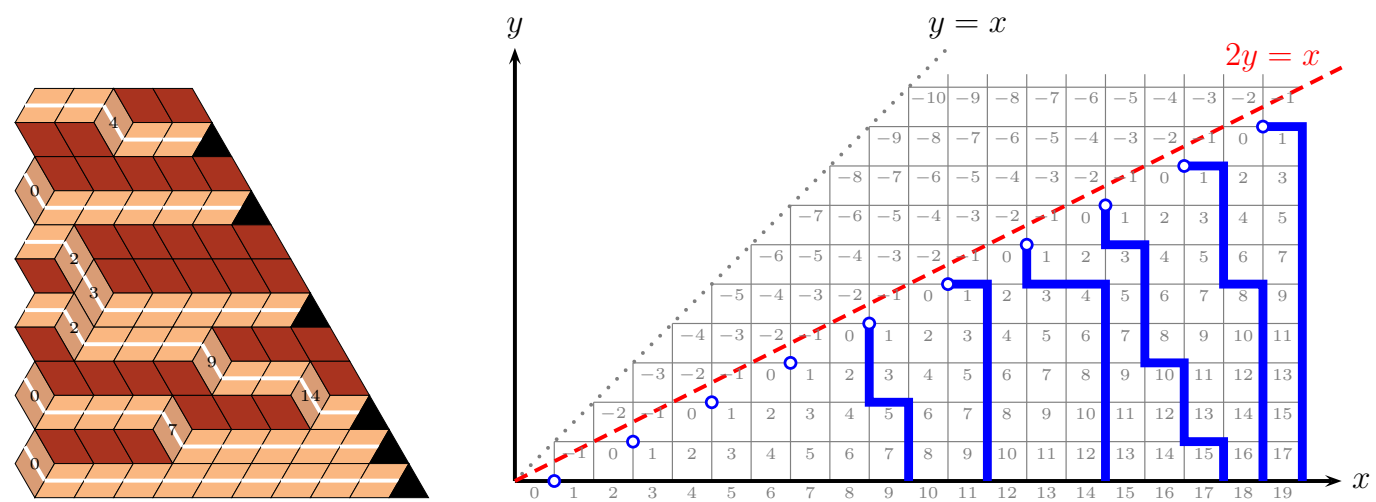

Figure 2: Left: The labeled tiling in the left picture is the same as in [4, Figure 1.5.b]. Note that the same picture, but with all labels of vertical lozenges increased by 1 , also corresponds to a dented half hexagon of the same height 12, but width 9 . This would give the labeled tiling in [4, Figure 1.5.a], and the family of nonintersecting lattice paths corresponding bijectively to this "relabelled" tiling is shown in the right picture. Right: A lozenge tiling of a dented quarter hexagon (basically, the "half of a half hexagon" of width $x=8$ and height 12), and the family of nonintersecting lattice paths corresponding bijectively to this tiling, but with all labels of vertical lozenges increased by 1.

\subsubsection{Apply the Lindström-Gessel-Viennot argument}

By the well-known Lindström-Gessel-Viennot argument [3,5], the generating function of all families of nonintersecting lattice paths can be written as a determinant of generating functions of (maybe intersecting) lattice paths. In our case, the generating function of all nonintersecting lattice paths starting at initial points $\left(a_{i}, a_{i}\right), i=1, \ldots n$, and ending at terminal points $\left(c_{j}, 0\right), j=1, \ldots n$, is given as

$$
\operatorname{det}\left(\overline{\mathfrak{g} f}\left(a_{i}, c_{j}\right)\right)_{i, j=1}^{n} .
$$

Using (8) and the multilinearity of the determinant, we obtain for all $n \in \mathbb{N}$ and all $n$-tuples $\left(a_{1}<a_{2}<\cdots<a_{n}\right)$ and $\left(c_{1}<c_{2}<\cdots<c_{n}\right)$ with $a_{i} \leq c_{i}, 1 \leq i \leq n$ :

$$
\frac{\operatorname{det}\left(\overline{\mathfrak{g} f}\left(a_{i}+d, c_{j}+d\right)\right)_{i, j=1}^{n}}{\operatorname{det}\left(\overline{\mathfrak{g} f}\left(a_{i}, c_{j}\right)\right)_{i, j=1}^{n}}=\prod_{m=1}^{n}\left(\frac{\left(q^{2 d+2} ; q^{2}\right)_{c_{m}}}{q^{d c_{m}} \cdot\left(q^{2} ; q^{2}\right)_{c_{m}}} \cdot \frac{q^{d a_{m}} \cdot\left(q^{2} ; q^{2}\right)_{a_{m}}}{\left(q^{2 d+2} ; q^{2}\right)_{a_{m}}}\right) .
$$

By the weight-preserving bijection between lozenge tilings and families of nonintersecting lattice paths, the quotient on the left hand side of (9) equals the quotient on the left hand side of (1) (after "translating" the notation in Theorem 2.1 to the "lattice path language"). So this argument reproves Lai's observation [4, Theorem 1.1]. (Basically the same simple approach, but restricted to mere enumeration, is contained in a recent preprint of Condon [2].)

\section{Quarter hexagons}

We now turn to the second type of regions considered by Lai, namely dented quarter hexagons (see the left picture in Figure 2, which shows a lozenge tiling of a dented quarter hexagon of width 8 and height 12). Again we translate lozenge tilings to nonintersecting lattice paths (as we did for dented half hexagons; see the right picture in Figure 2).

We see that lozenge tilings of a dented quarter hexagon correspond to families of nonintersecting lattice paths

- with initial points $(2 b, b)$ and terminal points $(c, 0)$ for the case of Lai's weight $w_{q}^{\prime}$, where $2 b \geq c \geq 0$,

- with initial points $(2 b+1, b)$ and terminal points $(c+1,0)$ for the case of Lai's weight $w_{q}$ (see the right picture in Figure 2, where the same tiling as in the left picture in Figure 2 is considered, but with labels of all vertical lozenges increased by 1 . This "shift of labels" implies starting points $(2 b+1, b)$ for the lattice paths), where $2 b \geq c \geq 0$.

Observe that for $X=Y=1$ we may rewrite (3) as follows (using again notation (6)):

$$
\left.\mathfrak{g}(a, b, c, d)\right|_{X=Y=1}=2^{a-c} q^{\frac{1}{2}(a-c)(a+c-1-4 d)} \frac{\left(-q^{2 a-2 b-2 d} ; q^{2}\right)_{c-a}\left(q^{2 b-2 d+2} ; q^{2}\right)_{c-a}}{\left(q^{2} ; q^{2}\right)_{c-a}} .
$$


So defining

we obtain from (10):

$$
\widehat{\mathfrak{g} \mathfrak{f}}(b, c):=\left.\mathfrak{g} \mathfrak{f}(2 b+1, b, c, 0)\right|_{X=Y=1}
$$

$$
\widehat{\mathfrak{g} \mathfrak{f}}(b, c)=2^{2 b+1-c} q^{b(2 b+1)-\frac{1}{2}(c-1) c} \frac{\left(q^{4 b+4} ; q^{4}\right)_{c-2 b-1}}{\left(q^{2} ; q^{2}\right)_{c-2 b-1}} .
$$

Note that by $(7), \widehat{\mathfrak{g} f}(b, c)=0$ for $c<2 b+1$.

Lai's "changing the width" of quartered hexagons corresponds bijectively to replacing $b \rightarrow b+x$ and $c \rightarrow c+2 x$ for some integer $x \geq-b$.

Remark 3.1. We shall not consider Lai's second weight $w^{\prime}$ here, which led to [4, Theorem 1.4]. The generating function of lattice paths corresponding to the weight $w^{\prime}$ can be expressed as

$$
\widehat{\mathfrak{g f} f^{\prime}}(b, c):=\left.\frac{1}{2} \mathfrak{g} \mathfrak{f}(2 b+1, b, c, 0)\right|_{X=Y=1}+\left.\mathfrak{g} \mathfrak{f}(2 b, b-1, c, 0)\right|_{X=Y=1}
$$

which by (10) can be rewritten as

$$
\widehat{\mathfrak{g f}^{\prime}}(b, c)=\frac{2^{2 b-c} q^{b(2 b-1)-\frac{1}{2}(c-1) c}\left(1-q^{2 c}\right)}{1-q^{4 b}} \frac{\left(q^{4 b+4} ; q^{4}\right)_{c-2 b}}{\left(q^{2} ; q^{2}\right)_{c-2 b}} .
$$

Note that by $(7), \widehat{\mathfrak{g f}^{\prime}}(b, c)=0$ for $c<2 b$.

From (11) we see that the generating function of all lattice paths with initial point $(2 x+1, x)$ and terminal point $(2 x+a, 0)$ is zero for $a<1$, otherwise it is

$$
\frac{2^{1-a} q^{-2 a x-\frac{1}{2}(a-1) a+2 x}\left(q^{4 x+4} ; q^{4}\right) a-1}{\left(q^{2} ; q^{2}\right) a-1} .
$$

Hence the generating function of all families of $m$ nonintersecting lattice paths with

- initial points $(2(x+i-1)+1, x+i-1), i=1,2, \ldots m$

- and terminal points $\left(2 x+a_{j}, 0\right), j=1,2, \ldots m$,

where $\mathbf{a}=\left(a_{j}\right)_{j=1}^{m}$ is an increasing sequence of nonnegative integers, is given as

$$
\begin{aligned}
2^{m^{2}-\sum_{j=1}^{m} a_{j}} q^{\frac{1}{2} \sum_{j=1}^{m}\left(a_{j}-a_{j}^{2}\right)+\frac{1}{6}\left(4 m^{3}-3 m^{2}-m\right)}\left(q^{2 x}\right)^{m^{2}-\sum_{j=1}^{m} a_{j}} & \times \operatorname{det}\left(\left[a_{j} \geq 2 i-1\right] \cdot \frac{\left(q^{4 i+4 x} ; q^{4}\right)_{a_{j}+1-2 i}}{\left(q^{2} ; q^{2}\right)_{a_{j}+1-2 i}}\right)_{i, j=1}^{m}
\end{aligned}
$$

Here, we used Iverson's bracket

$$
[\text { some assertion }]= \begin{cases}1 & : \text { if the assertion is true } \\ 0 & : \text { otherwise }\end{cases}
$$

to point out the obvious condition that a lattice path cannot have its end point to the left of its starting point; but note that by (7), we could also omit Iverson's bracket.

Clearly, the crucial point in (12) is the determinant; observe that it is zero if and only if $a_{j}<2 j-1$ for some $j, 1 \leq j \leq m$.

\section{The determinant appearing for dented quarter hexa- gons}

This section is devoted to the determinant in (12). We shall state and prove an identity regarding this determinant, from which Lai's Theorem 1.3 in [4] (restated as Theorem 2.2 in this paper) follows immediately.

Observe that the generating function (12) makes sense for arbitrary strictly increasing sequences $\left(a_{1}, a_{2}, \ldots, a_{m}\right)$ of positive integers, but for nonintersecting lattice paths corresponding to lozenge tilings of dented quarter hexagons, we must have the additional condition $a_{m} \leq 2 m$ (see the left picture in Figure 2), and if the generating function is non-zero, then we must also have $2 i-1 \leq a_{i}$ for $i=1,2, \ldots, m$. 


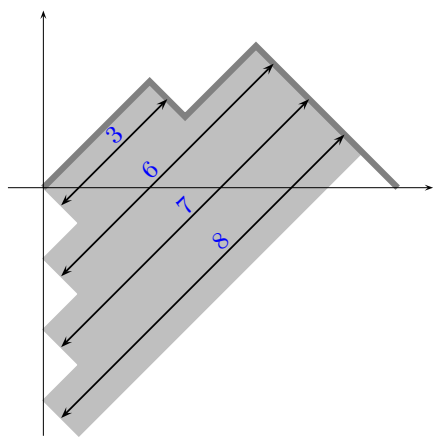

The picture illustrates the bijection for the admissible sequence $\mathbf{a}=(3,6,7,8)$ of length 4 and a Dyck path of length $2 \cdot 5=10$. For every down-step of the Dyck path except the last, draw a rectangle with one side equal to this down-step and one corner "leaning against the vertical axis"; the heights of these rectangles give an admissible sequence; and any admissible sequence "encodes" a Dyck path.

Figure 3: Illustration of the bijection between Dyck paths of length $2 m$ and admissible sequences of length $m-1$.

Definition 4.1. We call a finite sequence of integers $\mathbf{a}=\left(a_{1}, a_{2}, \ldots, a_{m}\right)$ admissible if it is strictly increasing and has the additional property

$$
2 i-1 \leq a_{i} \leq 2 m \text { for all } i, 1 \leq i \leq m \text {. }
$$

We call an admissible sequence irreducible if it obeys the stricter condition

$$
2 i+1 \leq a_{i} \leq 2 m \text { for all } i, 1 \leq i<m, \text { and } a_{m}=2 m
$$

Remark 4.1. Admissible sequences are yet another type of objects enumerated by the ubiquitous Catalannumbers: The number of admissible sequences of length $m-1$ is the Catalan number $C_{m}=\frac{1}{m+1}\left(\begin{array}{c}2 m \\ m\end{array}\right)$. One way to prove this is by giving a simple bijection with Dyck paths of length $2 m$ (i.e., with lattice paths from (0,0) to $(2 \mathrm{~m}, 0)$ which consist of diagonal upwards and downwards steps, but never fall below level 0), which can be easily "seen" by just looking at a picture; see Figure 3.

Theorem 4.1. Let $\mathbf{a}=\left(a_{1}, a_{2}, \ldots, a_{m}\right)$ be an admissible sequence, and define two $m \times m-m a t r i c e s \mathrm{M}(\mathbf{a}, x)$ and $\mathrm{S}(\mathbf{a})$ as follows:

$$
\mathrm{M}(\mathbf{a}, x):=\left(\frac{\left(q^{2 i+2 x} ; q^{2}\right)_{a_{j}+1-2 i}}{(q ; q)_{a_{j}+1-2 i}}\right)_{i, j=1}^{m} \text { and } \mathrm{S}(\mathbf{a}):=\left(\frac{1}{(q ; q)_{a_{j}+1-2 i}}\right)_{i, j=1}^{m}
$$

(Note that the entries in (13) are zero whenever $a_{j}<2 i-1$.)

Then we have

$$
\operatorname{det}(\mathrm{M}(\mathbf{a}, x))=\left(\prod_{k=1}^{m}\left(q^{2 x+2 k} ; q\right)_{a_{k}-2 k+1}\right) \operatorname{det}(\mathrm{S}(\mathbf{a})) .
$$

Observe that substituting $q \rightarrow q^{2}$ in (14) gives precisely the determinant in (12), so by the weight-preserving bijection between lozenge tilings and families of nonintersecting lattice paths, equation (14) yields Lai's observation [4, Theorem 1.3], that the quotient of two generating functions (12) with width parameters $x_{1}, x_{2}$ factors nicely.

Remark 4.2. The condition $a_{m} \leq 2 m$ in Theorem 4.1 is crucial; otherwise the assertion is wrong, already for $m=1$.

\subsection{The proof of Theorem 4.1}

This section is devoted to the proof of Theorem 4.1. The proof is rather long and consists of several steps, which we shall present in separate subsections.

\subsubsection{Reduce the general case to the irreducible case}

Observe that if there is some $1 \leq k<m$ such that $a_{k} \leq 2 k$, then the $(i, j)$-entry of $\mathrm{M}(\mathbf{a}, x)$ is zero for $i>k$ and $j<k$, so the determinant can be written as the product of two minors consisting 
- of the first $k$ rows and columns of $\mathrm{M}(\mathbf{a}, x)$,

- and of the last $(m-k)$ rows and columns of $\mathrm{M}(\mathbf{a}, x)$,

respectively. It is easy to see that these minors correspond to the lists

- $\mathbf{a}^{\prime}=\left(a_{1}, \ldots, a_{k}\right)$

- and $\mathbf{a}^{\prime \prime}=\left(a_{k+1}-2 k, \ldots, a_{m}-2 k\right)$,

which gives the following factorization:

$$
\operatorname{det}(\mathrm{M}(\mathbf{a}, x))=\operatorname{det}\left(\mathrm{M}\left(\mathbf{a}^{\prime}, x\right)\right) \cdot \operatorname{det}\left(\mathrm{M}\left(\mathbf{a}^{\prime \prime}, x+k\right)\right) .
$$

Both sequences $\mathbf{a}^{\prime}$ and $\mathbf{a}^{\prime \prime}$ are admissible, and if we manage to prove Theorem 4.1 for the irreducible case

$$
a_{k} \geq 2 k+1 \text { for all } k=1, \ldots, m-1,
$$

it is easy to see that the factorization above gives the general assertion (14) for all admissible sequences (by induction on the number of "irreducible factors").

Note that an irreducible sequence a of length $m$ necessarly ends with

$$
\mathbf{a}=\left(\ldots, a_{m-1}=2 m-1, a_{m}=2 m\right) .
$$

\subsubsection{Pull out common factors from rows and columns}

We may rewrite the factors of the product in (14) as follows

$$
\prod_{k=1}^{m}\left(q^{2 x+2 k} ; q\right)_{a_{k}-2 k+1}=\frac{\prod_{j=1}^{m}\left(q^{2 x} ; q\right)_{a_{j}+1}}{\prod_{i=1}^{m}\left(q^{2 x} ; q\right)_{2 i}}
$$

and pull

- the numerator $\left(q^{2 x} ; q\right)_{a_{j}+1}$ out from column $j, j=1, \ldots, m$,

- and the denominator $\left(q^{2 x} ; q\right)_{2 i}$ out from row $i, i=1 \ldots, m$

in $\mathrm{M}(\mathbf{a}, x)$. This operation gives a new $m \times m$-matrix $\mathrm{M}^{\prime}(\mathbf{a}, x)$ with $(i, j)$-entry

$$
\frac{\left(q^{2 x} ; q\right)_{2 i}\left(q^{2 i+2 x} ; q^{2}\right)_{a_{j}+1-2 i}}{\left(q^{2 x} ; q\right)_{a_{j}+1}(q ; q)_{a_{j}+1-2 i}}=\frac{\left(q^{2 x+2 i} ; q^{2}\right)_{a_{j}+1-2 i}}{\left(q^{2 x+2 i} ; q\right)_{a_{j}+1-2 i}(q ; q)_{a_{j}+1-2 i}},
$$

and (14) clearly is equivalent to

$$
\operatorname{det}\left(\mathrm{M}^{\prime}(\mathbf{a}, x)\right)=\operatorname{det}(\mathrm{S}(\mathbf{a}))
$$

\subsubsection{Method of proof: Triangulate the modified matrix $\mathrm{M}^{\prime}(\mathrm{a}, x)$}

We want to show that the modified matrix $\mathrm{M}^{\prime}(\mathbf{a}, x)$ can be triangulated by a lower triangular matrix $\mathrm{F}(\mathbf{a})$ with entries from the field $\mathbb{C}(q)$ of rational functions in $q$, which we denote by $\mathbb{F}$.

Lemma 4.1. Let $\mathbf{a}$ be an irreducible sequence, and consider $\mathrm{M}^{\prime}(\mathbf{a}, x)$ as defined in (15). Then there exists a lower triangular $m \times m$-matrix $\mathrm{F}(\mathbf{a})$ whose entries are constants from $\mathbb{F}$ (i.e., the entries do not depend on $x$ ) with all entries on the main diagonal equal to 1 , such that the matrix product

$$
\mathrm{M}^{\prime}(\mathbf{a}, x) \cdot \mathrm{F}(\mathbf{a})
$$

is upper triangular, with constants from $\mathbb{F}$ on its main diagonal.

If we manage to prove Lemma 4.1 , then by the multiplicativity of the determinant, we would obtain that

$$
\operatorname{det}\left(\mathrm{M}^{\prime}(\mathbf{a}, x) \cdot \mathrm{F}(\mathbf{a})\right)=\operatorname{det}\left(\mathrm{M}^{\prime}(\mathbf{a}, x)\right) \cdot \operatorname{det}(\mathrm{F}(\mathbf{a}))=\operatorname{det}\left(\mathrm{M}^{\prime}(\mathbf{a}, x)\right),
$$

and this determinant is equal to the product of the entries on the main diagonal of (17); so, in particular, it does not depend on $x$. (This would already be sufficient to deduce Lai's observation concerning the quotient of generating functions [4, Theorem 1.3].) Moreover, for $q \in \mathbb{C}$ with $|q|<1$, (16) would also hold in the limit $x \rightarrow \infty$, so (14) follows since

$$
\lim _{x \rightarrow \infty} \frac{\left(q^{2 x+2 i} ; q^{2}\right)_{a_{j}+1-2 i}}{\left(q^{2 x+2 i} ; q\right)_{a_{j}+1-2 i}(q ; q)_{a_{j}+1-2 i}}=\frac{1}{(q ; q)_{a_{j}+1-2 i}} \text { for }|q|<1 .
$$

Thus the proof of Theorem 4.1 will be complete if we can prove Lemma 4.1. 


\subsection{Proof of Lemma 4.1}

The following subsections are devoted to the proof of Lemma 4.1 .

\subsubsection{Find the matrix $\mathrm{F}(\mathrm{a})$ by solving systems of linear equations}

Finding the triangulating matrix $\mathrm{F}(\mathbf{a})$ amounts to solving $(m-1)$ systems of linear equations, as the following considerations show. For some matrix A, let us denote by $[\mathrm{A}]_{i \geq s, j \geq t}$ the submatrix of A consisting

- of all rows $i \geq s$

- and all columns $j \geq t$.

The $k$-th column vector in a lower triangular $m \times m$-matrix has, by definition, zero entries at all positions $j<k$, so determining the sub-vector $\mathbf{v}_{k}$ corresponding to the "interesting" entries of this column vector in the lower triangular $m \times m$-matrix $\mathrm{F}(\mathbf{a})$ amounts to finding a solution of the homogeneous system of linear equations

$$
\left[\mathrm{M}^{\prime}(\mathbf{a}, x)\right]_{i \geq k+1, j \geq k} \cdot \mathbf{v}_{k}=\mathbf{0}
$$

which has the following additional properties:

1. the entries in $\mathbf{v}_{k}$ are elements of $\mathbb{F}$ which do not depend on $x$. We call such solution $x$-invariant,

2. the first entry of $\mathbf{v}_{k}$ is not zero, whence we can divide $\mathbf{v}_{k}$ by this first entry (so the lower triangular matrix $\mathrm{F}$ (a) would have entries equal to 1 on its main diagonal, as desired),

3. the inner product of the first row of $\left[\mathrm{M}^{\prime}(\mathbf{a}, x)\right]_{i \geq k, j \geq k}$ with $\mathbf{v}_{k}$ is in $\mathbb{F}$ and does not depend on $x$, too.

Clearly, we have to find such solutions for $k=1,2, \ldots, m-1$.

\subsubsection{Reduce to a single system of linear equations}

It is easy to see that the submatrix $\left[\mathrm{M}^{\prime}(\mathbf{a}, x)\right]_{i \geq 2, j \geq 2}$ is precisely the $(m-1) \times(m-1)-$ matrix $\mathrm{M}^{\prime}(\check{\mathbf{a}}, x+1)$, where

$$
\check{\mathbf{a}}=\left(a_{2}-2, a_{3}-2, \ldots, a_{m}-2\right) .
$$

Hence it suffices to consider only the first column vector $\mathbf{v}_{1}$ of the matrix $\mathrm{F}(\mathbf{a})$. This means that we have to show that the system of equations

$$
\left[\mathrm{M}^{\prime}(\mathbf{a}, x)\right]_{i \geq 2, j \geq 1} \cdot \mathbf{v}_{1}=\mathbf{0}
$$

has a solution with the additional properties 1, 2 and 3 from above.

\subsubsection{Express the system of linear equations more conveniently}

Observe that we can express property 3 more conveniently: Prepend 1 to the irreducible sequence a, i.e., consider the (admissible!) sequence

$$
\mathbf{a}^{\star}:=\left(1, a_{1}, \ldots, a_{m}\right)
$$

(so $a_{1}^{\star}=1$ and $a_{k}^{\star}=a_{k-1}$ for $\left.2 \leq k \leq m+1\right)$, and define the $m \times(m+1)$-matrix $\mathrm{M}^{\prime}\left(\mathbf{a}^{\star}, x\right)$ with entries as in (15), i.e.:

$$
\mathrm{M}^{\prime}\left(\mathbf{a}^{\star}, x\right)_{i, j}=\frac{\left(q^{2 x+2 i} ; q^{2}\right)_{a_{j}^{\star}+1-2 i}}{\left(q^{2 x+2 i} ; q\right)_{a_{j}^{\star}+1-2 i}(q ; q)_{a_{j}^{\star}+1-2 i}} .
$$

Note that $\mathrm{M}^{\prime}\left(\mathbf{a}^{\star}, x\right)_{i, 1}=[i=1]$. Hence an $x$-invariant solution of

$$
\mathrm{M}^{\prime}\left(\mathbf{a}^{\star}, x\right) \cdot \mathbf{v}_{1}^{\star}=\mathbf{0}
$$

gives an $x$-invariant solution $\mathbf{v}_{1}$ of (18) with property 3 , by simply deleting the first entry of $\mathbf{v}_{1}^{\star}$ :

$$
\mathbf{v}_{1}^{\star}=\left(v_{0}, v_{1}, \ldots, v_{m}\right) \mapsto \mathbf{v}_{1}=\left(v_{1}, \ldots, v_{m}\right) .
$$

Assume that we can show that (20) has a non-trivial $x$-invariant solution. Then such solution would determine a matrix $\mathrm{F}(\mathbf{a})$ which might have zeros on the main diagonal; but this cannot happen since we know that $\operatorname{det}\left(\mathrm{M}^{\prime}(\mathbf{a}, x)\right) \neq 0$ (as it corresponds to the generating function of a non-empty set of nonintersecting lattice paths). This means that such solutions "automatically" have property 2: So the proof of Lemma 4.1 boils down to showing that $(20)$ has a non-trivial $x$-invariant solution. 


\subsubsection{Divide all equations by the coefficient corresponding to the last column}

The system (20) features $m$ equations for $m+1$ variables, so for every fixed $x$ we expect a solutions space of dimension $\geq 1$. As pointed out above, element $a_{m}$ equals $2 m$ in an irreducible sequence of length $m$. By dividing the $i$-th equation (i.e., row $i$ of the matrix $\mathrm{M}^{\prime}\left(\mathbf{a}^{\star}, x\right)$ ) by the (non-zero) coefficient corresponding to $a_{m+1}^{\star}=a_{m}=2 m$, which is

$$
\frac{\left(q^{2 x+2 i} ; q^{2}\right)_{2 m+1-2 i}}{\left(q^{2 x+2 i} ; q\right)_{2 m+1-2 i}(q ; q)_{2 m+1-2 i}},
$$

we do not change the space of solutions, but obtain a modified coefficient matrix $\mathrm{M}^{\prime \prime}\left(\mathbf{a}^{\star}, x\right)$ with $(i, j)$-entry

$$
\frac{\left(q^{2 x+2 i} ; q^{2}\right)_{a_{j}+1-2 i}\left(q^{2 x+2 i} ; q\right)_{2 m+1-2 i}(q ; q)_{2 m+1-2 i}}{\left(q^{2 x+2 i} ; q^{2}\right)_{2 m+1-2 i}\left(q^{2 x+2 i} ; q\right)_{a_{j}+1-2 i}(q ; q)_{a_{j}+1-2 i}}=\frac{\left(q^{2+a_{j}-2 i} ; q\right)_{2 m-a_{j}}\left(q^{1+a_{j}+2 x} ; q\right)_{2 m-a_{j}}}{\left(q^{2+2 a_{j}+2 x-2 i} ; q^{2}\right)_{2 m-a_{j}}} .
$$

So we have to find an $x$-invariant solution of the system of homogeneous equations

$$
\mathrm{M}^{\prime \prime}\left(\mathbf{a}^{\star}, x\right) \cdot \mathbf{v}_{1}^{\star}=\mathbf{0} .
$$

\subsubsection{Consider a system of linear equations with more variables}

It is convenient to view the coefficient matrix $\mathrm{M}^{\prime \prime}\left(\mathbf{a}^{\star}, x\right)$ as the submatrix corresponding to columns $a_{1}^{\star}, a_{2}^{\star}, \ldots, a_{m+1}^{\star}$ of the $m \times 2 m$-matrix with $(i, j)$-entry

$$
\frac{\left(q^{2+j-2 i} ; q\right)_{2 m-j}\left(q^{1+j+2 x} ; q\right)_{2 m-j}}{\left(q^{2+2 j+2 x-2 i} ; q^{2}\right)_{2 m-j}},
$$

and to reverse the order of columns, i.e., to consider the $m \times 2 m$-matrix $\mathrm{M}^{\prime \prime \prime}(m, x)$ with $(i, j)$-entry

$$
\mathbf{e}(m, i, j, x):=\frac{\left(q^{2(m-i+1)-j} ; q\right)_{j}\left(q^{2(m+x)-j+1} ; q\right)}{\left(q^{2(2 m+x-i+1)-2 j} ; q^{2}\right)_{j}},
$$

where $1 \leq i \leq m$ and $0 \leq j \leq 2 m-1$.

I.e., the $m+1$ columns of $\mathrm{M}^{\prime \prime}\left(\mathbf{a}^{\star}, x\right)$ correspond to the columns

$$
\bar{a}_{j}=2 m-a_{m+1-j}^{\star} \text { for } j=0,1, \ldots, m,
$$

in $\mathrm{M}^{\prime \prime \prime}(m, x)$, and the sequence $\overline{\mathbf{a}}=\left(\bar{a}_{0}, \bar{a}_{1}, \ldots, \bar{a}_{m}\right)$ is strictly increasing with the additional properties

$$
\bar{a}_{j} \leq 2 j-1 \text { for } j=1,2, \ldots, m \text { (since } \mathbf{a}^{\star} \text { is admissible), }
$$

and

$$
\bar{a}_{0}=2 m-a_{m+1}^{\star}=0, \bar{a}_{1}=2 m-a_{m}^{\star}=1 \text { and } \bar{a}_{m}=2 m-a_{1}^{\star}=2 m-1 .
$$

We call a sequence with these properties reversed admissibly.

Observe that the factor $\left(q^{2(m-i+1)-j} ; q\right)$ in $(23)$ is zero for $j \geq 2(m-i+1)$, hence the matrix $\mathrm{M}^{\prime \prime \prime}(m, x)$ has a "staircase shape", with all entries of row $i$ equal to zero for $j \geq 2(m-i+1)$. To illustrate this, we present $\mathrm{M}^{\prime \prime \prime}(3, x)$ (after expansion of the $q$-Pochhammer symbols involving the parameter $x$ and cancellation; for typesetting reasons, we give the transpose of this matrix):

$$
\left(\mathrm{M}^{\prime \prime \prime}(3, x)\right)^{T}=\left(\begin{array}{ccc}
1 & 1 & 1 \\
\frac{\left(q^{5} ; q\right)_{1}\left(1-q^{2 x+6}\right)}{1-q^{2 x+10}} & \frac{\left(q^{3} ; q\right)_{1}\left(1-q^{2 x+6}\right)}{1-q^{2 x+8}} & 1-q \\
\frac{\left(q^{4} ; q\right)_{2}\left(1-q^{2 x+5}\right)\left(1-q^{2 x+6}\right)}{\left(1-q^{2 x+8}\right)\left(1-q^{2 x+10}\right)} & \frac{\left(q^{2} ; q\right)_{2}\left(1-q^{2 x+5}\right)}{1-q^{2 x+8}} & 0 \\
\frac{\left(q^{3} ; q\right)_{3}\left(1-q^{2 x+4}\right)\left(1-q^{2 x+5}\right)}{\left(1-q^{2 x+8}\right)\left(1-q^{2 x+10}\right)} & \frac{(q ; q)_{3}\left(1-q^{2 x+5}\right)}{1-q^{2 x+8}} & 0 \\
\frac{\left(q^{2} ; q\right)_{4}\left(1-q^{2 x+3}\right)\left(1-q^{2 x+5}\right)}{\left(1-q^{2 x+8}\right)\left(1-q^{2 x+10}\right)} & 0 & 0 \\
\frac{(q ; q)_{5}\left(1-q^{2 x+3}\right)\left(1-q^{2 x+5}\right)}{\left(1-q^{2 x+8}\right)\left(1-q^{2 x+10}\right)} & 0 & 0
\end{array}\right)
$$




\subsubsection{Make a useful observation concerning (23)}

The attentive reader may have noticed a phenomenon in the example (24). The number of factors in the denominator is not increasing all the way, but apparently becomes constant from $j=m-i$ on. This is no coincidence, but due to certain cancellations. Observe that the factors depending on $x$ in the numerator of (23) end with $\left(1-q^{2 m+2 x}\right)$, and from $j=m-i+1+t$ on $(t=0,1, \ldots)$, the "additional factors" $\left(1-q^{2 m+2 x-2 t}\right)$ appearing in the denominator cancel out with corresponding factors in the numerator, such that in the last two entries of each row only factors with odd " $x$-depending $q$-exponents" $\left(1-q^{2 x+2 t-1}\right)$ survive the cancellation in the numerator; moreover, the last entry is $(1-q)$-times the next-to-last entry. Altogether, this implies. If we multiply every row $i$ of $\mathrm{M}^{\prime \prime \prime}(m, x)$ by the denominator of its last non-zero entry

$$
\left(q^{2 x+2 m+2} ; q^{2}\right)_{m-i},
$$

then the $(i, j)$-entry of the resulting matrix is equal to $\left(q^{2(m-i+1)-j} ; q\right)$ times a product of $(m-i)$ factors of the form $\left(1-q^{2 x+y}\right)$, i.e.,

$$
\mathrm{M}^{\prime \prime \prime}(m, x)_{i, j} \cdot\left(q^{2 x+2 m+2} ; q^{2}\right)_{m-i}=\left(q^{2(m-i+1)-j} ; q\right)_{j} \cdot \prod_{k=1}^{m-i}\left(1-q^{2 x+y_{j, k}}\right)
$$

for certain integers $y_{j, k}$.

\subsubsection{Claim: The first homogeneous equation of (22) actually has a non-trivial $x$-invariant solution}

It might not be clear at first sight that there is a non-trivial $x$-invariant solution at all; even for a single equation of (22): In order to show that this is indeed the case, we state and prove a little Lemma.

Recall the definition of the $q$-binomial coefficient

$$
\left[\begin{array}{l}
n \\
k
\end{array}\right]_{q}:=\frac{(q ; q)_{n}}{(q ; q)_{k}(q ; q)_{n-k}}
$$

for non-negative integers $n, k$ with $0 \leq k \leq n$, and the well-known recursions

$$
\begin{aligned}
& {\left[\begin{array}{l}
n \\
k
\end{array}\right]_{q}=\left[\begin{array}{l}
n-1 \\
k-1
\end{array}\right]_{q}+q^{k}\left[\begin{array}{c}
n-1 \\
k
\end{array}\right]_{q},} \\
& {\left[\begin{array}{l}
n \\
k
\end{array}\right]_{q}=q^{n-k}\left[\begin{array}{l}
n-1 \\
k-1
\end{array}\right]_{q}+\left[\begin{array}{c}
n-1 \\
k
\end{array}\right]_{q} .}
\end{aligned}
$$

An easy consequence of these recursions is the well-known identity

$$
\sum_{k=0}^{n}(-1)^{k} q^{\frac{k(k+1)}{2}-k n}\left[\begin{array}{l}
n \\
k
\end{array}\right]_{q}=[n=0] .
$$

Now define

$$
e(n, k):=\frac{k(k+1)}{2}-k n
$$

and observe that (29) can be generalized as follows:

Lemma 4.2. Let $n, r$ be positive integers, and let $\left(\gamma_{1}, \gamma_{2}, \ldots, \gamma_{n-r}\right)$ be an (arbitrary) sequence of $n-r$ complex numbers. Then we have

$$
\sum_{k=0}^{n}(-1)^{k} q^{e(n, k)}\left[\begin{array}{l}
n \\
k
\end{array}\right] \prod_{q}^{n-r}\left(1-\gamma_{i} \cdot q^{k}\right)=0 .
$$

Proof. Let $r$ be arbitrary but fixed, and proceed by induction on $n$. Clearly, for $n \leq r$ the statement is true, since it is equivalent to (29) in this case.

For the inductive step $(n-1) \rightarrow n$, we set $f:=\prod_{i=1}^{n-1-r}\left(1-\gamma_{i} \cdot q^{k}\right)$ and use the recursions (27) and (28) to rewrite the unsigned $k$-th summand in (30) as

$$
q^{e(n, k)}\left(\left(\left[\begin{array}{c}
n-1 \\
k
\end{array}\right]_{q} q^{k}+\left[\begin{array}{l}
n-1 \\
k-1
\end{array}\right]_{q}\right)-\gamma_{n-r} \cdot q^{k}\left(\left[\begin{array}{c}
n-1 \\
k
\end{array}\right]_{q}+\left[\begin{array}{l}
n-1 \\
k-1
\end{array}\right]_{q} q^{n-k}\right)\right) f .
$$


Using the obvious relations

$$
e(n, k)+k=e(n-1, k) \text { and } e(n, k)+n-1=e(n-1, k-1)
$$

we may rewrite this as

$$
\left(\left[\begin{array}{c}
n-1 \\
k
\end{array}\right]_{q} q^{e(n-1, k)}+q^{1-n}\left[\begin{array}{l}
n-1 \\
k-1
\end{array}\right]_{q} q^{e(n-1, k-1)}\right) f-\gamma_{n-r}\left(\left[\begin{array}{c}
n-1 \\
k
\end{array}\right]_{q} q^{e(n-1, k)}+q\left[\begin{array}{l}
n-1 \\
k-1
\end{array}\right]_{q} q^{e(n-1, k-1)}\right) f
$$

and the assertion follows by induction.

Remark 4.3. Christian Krattenthaler pointed out to me that Lemma 4.2 is, in fact, a standard result of $q$-difference calculus: Note that the $q$-differential operator $D_{q}$

$$
D_{q} p(x)=\frac{p(q x)-p(x)}{(q-1) x}
$$

can be expressed in terms of the operators $I$ and $E_{q}$,

$$
I p(x):=p(x), E_{q} p(x):=p(q x),
$$

as

$$
D_{q}=\frac{E_{q}-I}{(q-1) x}
$$

Since

$$
D_{q} x^{0} \equiv 0 \text { and } D_{q} x^{n}=\frac{q^{n}-1}{q-1} x^{n-1} \text { for } n>0,
$$

$D_{q}$ decreases the degree (in $x$ ) of any polynomial, so

$$
D_{q}^{n} p \equiv 0
$$

for all polynomials $p$ of degree $<n$. Now observe that operators $A:=x^{-1} E_{q}$ and $B:=x^{-1} I$ fulfil

$$
B A=q A B,
$$

so

$$
D_{q}^{n}=\frac{1}{(q-1)^{n}}(A-B)^{n}=\sum_{k=0}^{n}(-1)^{n-k}\left[\begin{array}{l}
n \\
k
\end{array}\right]_{q} A^{k} B^{n-k}
$$

by Schützenberger's q-binomial Theorem [7]. Therefore, for all polynomials $p$ of degree $<n$ we have

$$
\begin{aligned}
0=(A-B)^{n} p(x) & =\sum_{k=0}^{n}(-1)^{n-k}\left[\begin{array}{l}
n \\
k
\end{array}\right]_{q}\left(x^{-1} E_{q}\right)^{k}\left(x^{-1}\right)^{n-k} p(x) \\
& =x^{-n} \sum_{k=0}^{n}(-1)^{n-k}\left[\begin{array}{l}
n \\
k
\end{array}\right]_{q} q^{-\left(\begin{array}{c}
k \\
2
\end{array}\right)-k(n-k)} E_{q}^{k} p(x) \\
& =x^{-n} \sum_{k=0}^{n}(-1)^{n-k}\left[\begin{array}{l}
n \\
k
\end{array}\right]_{q} q^{\left(\begin{array}{c}
k+1 \\
2
\end{array}\right)-k n} p\left(q^{k} x\right),
\end{aligned}
$$

which gives another proof of Lemma 4.2.

From Lemma 4.2 we can deduce immediately the following Corollary.

Corollary 4.1. Let $n, r$ be positive integers, and assume that for each for $j=1,2, \ldots, n+1$ we are given

- $n-r$ complex numbers $\gamma_{j, 1}, \gamma_{j, 2}, \ldots, \gamma_{j, n-r}$

- and a rational function $c_{j}=c_{j}(q)$ in $\mathbb{F}$.

Then any $n+1$ subsequent rows $i=i_{0}+1, i_{0}+2, \ldots, i_{0}+n+1$ (for arbitrary $i_{0} \in \mathbb{N}$ ) of the form

$$
\left(c_{j} \prod_{k=1}^{n-r}\left(1-\gamma_{j, k} q^{i}\right)\right)_{j=1}^{n+1}
$$


are not linearly independent over $\mathbb{F}$ (by Lemma 4.2), and therefore the same is true for the columns of the matrix

$$
\left(c_{j} \prod_{k=1}^{n-r}\left(1-\gamma_{j, k} q^{i}\right)\right)_{(i, j)=\left(i_{0}+1,1\right)}^{\left(i_{0}+n+1, n+1\right)}
$$

Hence the homogeneous system of linear equations corresponding to this matrix has a nontrivial solution $\mathbf{b}=\left(b_{1}, b_{2}, \ldots, b_{n+1}\right)$ in $\mathbb{F}$.

Since such solution $\mathbf{b}$ fulfils the $n$ subsequent equations corresponding to rows $i_{0}+2, i_{0}+3, \ldots, i_{0}+n+1$, it also fulfils the equation corresponding to row $i_{0}+n+2$ (since this row is a linear combination of its $n$ predecessors, by Lemma 4.2) and the equation corresponding to row $i_{0}+0$ (since this is a linear combination of its $n$ successors, again by Lemma 4.2).

In short, every solution $\mathbf{b}$ of the homogeneous system of linear equations corresponding to (32) fulfils the equations corresponding to rows of the form (31) for all $i \in \mathbb{N}$, and in that sense is $i$-invariant. Therefore we have for all complex numbers $q$ with $|q|<1$

$$
0=\lim _{i \rightarrow \infty} \sum_{j=1}^{n+1} c_{j}\left(\prod_{k=1}^{n-r}\left(1-\gamma_{j, k} q^{i}\right)\right) b_{j}=\sum_{j=1}^{n+1} c_{j} b_{j},
$$

so $\mathbf{b}$ is a solution to the simpler equation

$$
\sum_{j=1}^{n+1} c_{j} b_{j}=0
$$

Now observe that Corollary 4.1 applies to the first row of $\mathrm{M}^{\prime \prime}\left(\mathbf{a}^{\star}, x\right)$ after multiplication by the denominator of its last non-zero entry (as in $(25)$ ). The $j$-th entry in this row is equal to $\left(q^{2 m-j} ; q\right)$, times a product of $m-1$ factors of the form $\left(1-q^{2 x+y}\right)$, and the length of this row is $m+1$; hence we may consider the $m+1$ equations for $x, x+1 / 2, \ldots, x+m / 2$ and deduce that there exists an $x$-invariant solution for the first equation of (22).

\subsubsection{Make another useful observation concerning (23)}

We set $x=\frac{1}{2}-k$ in $\mathbf{e}(m, i, j, x)$ and observe:

$$
\begin{aligned}
\mathbf{e}\left(m, i, j, \frac{1}{2}-k\right) & =\frac{\left(q^{2(m-i+1)-j} ; q\right)_{j}\left(q^{2(m-k+1)-j} ; q\right)_{j}}{\left(q^{2\left(2 m-k+\frac{1}{2}-i+1\right)-2 j} ; q^{2}\right)_{j}} \\
& =\frac{\left(q^{2(m-k+1)-j} ; q\right)_{j}\left(q^{2\left(m+\left(\frac{1}{2}-i\right)\right)-j+1} ; q\right)_{j}}{\left(q^{2\left(2 m+\left(\frac{1}{2}-i\right)-k+1\right)-2 j} ; q^{2}\right)_{j}} \\
& =\mathbf{e}(m, k, j, 1 / 2-i) .
\end{aligned}
$$

Note that this means any $x$-invariant solution of the equation corresponding to row $k$ of $\mathrm{M}^{\prime \prime}\left(\mathbf{a}^{\star}, x\right)$ is also a solution of all rows $i \geq k$, but for fixed $x=\frac{1}{2}-k$.

\subsubsection{Put everything together}

For some subsequence $C \subseteq\{0,1, \ldots, 2 m-1\}$ of columns of $\mathrm{M}^{\prime \prime \prime}(m, x)$, we may consider the homogeneous system of linear equations corresponding to the submatrix of $\mathrm{M}^{\prime \prime \prime}(m, x)$ which consists of the columns in $C$. We call this system the equations corresponding to $C$, and if we consider only the last $k(1 \leq k \leq m)$ of these equations (corresponding to the submatrix of $\mathrm{M}^{\prime \prime \prime}(m, x)$ which consists of the rows $m-k+1, m-k+2, \ldots, m$ and the columns in $C$ ), we call this homogeneous system the last $k$ equations corresponding to $C$. For arbitrary but fixed $x$, the set of solutions of the last $k$ equations corresponding to some $C$ is a linear subspace of $\mathbb{F}^{|C|}$, which contains the set of $x$-invariant solutions, and the latter is a linear subspace of $\mathbb{F}^{|C|}$, too.

Recall that we have to show that the homogeneous equations corresponding to $\overline{\mathbf{a}}$ (which are equivalent to (22)) have a one-dimensional $x$-invariant space of solutions: We shall prove this by working our way up from the last row $m$ to the first.

More precisely, we claim that the last $k$ rows of the equations corresponding to $\overline{\mathbf{a}}$

- have an $x$-invariant solutions space whose dimension is equal

- to the number $l$ of elements $\bar{a}_{j}$ in $\overline{\mathbf{a}}$ such that $\bar{a}_{j} \leq 2 k-1$ 
$-\operatorname{minus} k$,

- and that there are no other solutions, that is, every solution (for arbitrary fixed $x$ ) is, in fact $x$-invariant.

Note that for $k \geq 1$ we have $l \geq k+1$ since $\bar{a}$ is reversed admissible, i.e.,

$$
\bar{a}_{0}=0 \text { and } \bar{a}_{j} \leq 2 j-1 \text { for } j=1,2, \ldots, m \text {; }
$$

so the (claimed) dimension $(l-k)$ of the solutions space is greater or equal to 1 .

The claim is immediately clear for $k=1$, since the (single) last equation corresponding to $\overline{\mathbf{a}}$ has only two non-zero coefficients for the two elements

$$
\bar{a}_{0}=0 \leq \bar{a}_{1}=1
$$

of $\overline{\mathbf{a}}$ which are less or equal to $2 k-1=1$, and this equation (see the last column of example (24), which shows the transposed matrix) has a 1 -dimensional solutions space, which is $x$-invariant since it does not contain $x$ at all:

$$
1 \cdot c_{0}+(1-q) \cdot c_{1}=0 \Longleftrightarrow\left(c_{0}, c_{1}\right) \in\{\lambda \cdot(q-1,1): \lambda \in \mathbb{C}\} .
$$

So assume we proved our claim for the last $k$ rows

$$
m-k+1, m-k+2, \ldots, m
$$

of the equations corresponding to $\overline{\mathbf{a}}$, and assume that for the next row $(m-k) \geq 1$ there are $l$ columns of $\overline{\mathbf{a}}$ which are $\leq 2(k+1)-1$. Now recall $(25)$, the entries of row $(m-k)$ are, after multiplication with the denominator of the entry $\mathrm{M}^{\prime \prime \prime}(m, x)_{m-k, 2(m-k)-1}$, equal to some element in $\mathbb{F}$ times a product of $k$ factors of the form $\left(1-q^{2 x+y}\right)$. So by Corollary 4.1 , row $(m-k)$ in the equations corresponding to

- the first $k+1$ elements $\bar{a}_{0}, \bar{a}_{1}, \ldots, \bar{a}_{k}$ of $\overline{\mathbf{a}}$,

- plus one of the $(l-(k+1)) \geq 1$ columns $\bar{a}_{j}, j>k$, which are less or equal to $2(k+1)-1$

has a non-trivial $x$-invariant solution, which we may view as an element in $\mathbb{F}^{m+1}$ (by "inserting" zeros in the solution vector for all coefficients corresponding to elements $\bar{a}_{i}$ with $i>k$ and $i \neq j$ ), and every linear combination of these $(l-(k+1))$ non-trivial $x$-invariant solutions (which clearly are linear independent in $\left.\mathbb{F}^{m+1}\right)$ is again an $x$-invariant solution, so the dimension of the linear space of $x$-invariant solutions is at least $(l-(k+1))$.

Now we combine two observations:

1. Since the first $k+1$ entries of $\overline{\mathbf{a}}$ are a reversed admissible sequence, the submatrix of $\mathrm{M}^{\prime \prime \prime}(m, x)$ consisting of

- the last $k+1$ rows

- and the columns indexed by the first $k+1$ elements $\bar{a}_{0}, \bar{a}_{1}, \ldots, \bar{a}_{k}$ of $\overline{\mathbf{a}}$

has determinant $\neq 0$ (since it is the generating function of a non-empty family of nonintersecting lattice paths). Hence the $k+1$ equations are linearly independent over $\mathbb{F}$, and the dimension of the solutions space for arbitrary, but fixed $x$ is $(l-(k+1))$. But this implies that all solutions (for any $x$ ) of the $(m-k)$-th equation corresponding to $\overline{\mathbf{a}}$ are, in fact, $x$-invariant.

2. By observation (33), for $i \leq k$ the following rows are identical:

- row $m-i$ for $x=\frac{1}{2}-(m-k)$,

- row $m-k$ for $x=\frac{1}{2}-(m-i)$.

This implies that each $x$-invariant solution of the $(m-k)$-th equation corresponding to $\overline{\mathbf{a}}$ is a solution of the last $k+1$ equations corresponding to $\overline{\mathbf{a}}$ for fixed $x=\frac{1}{2}-(m-k)$, and since we already know that all solutions (for any $x$ ) of the last $k$ equations are $x$-invariant, the same holds true for the last $k+1$ equations.

Altogether, this finishes the proof of Lemma 4.1, and thus of Theorem 4.1. 


\subsection{A simple algorithm for actually finding the solutions which give $\mathrm{F}(\mathrm{a})$ of Lemma 4.1}

For the homogeneous system of the $m$ linear independent equations in $2 m$ variables corresponding to the coefficient matrix $\mathrm{M}^{\prime \prime \prime}$

$$
\mathrm{M}^{\prime \prime \prime} \cdot \mathbf{c}=\mathbf{0},
$$

we expect a solutions space of dimension $m$. We claim that this solutions space is $x$-invariant and spanned by $m$ vectors $\mathbf{c}_{1}, \ldots, \mathbf{c}_{m}$, where

$$
\mathbf{c}_{1}=\left(1, \alpha_{1}, 0, \alpha_{3}, \ldots, 0, \alpha_{2 m-1}\right)
$$

equals the $2 m$ first elements of a certain infinite sequence in $\mathbb{F}$ (this sequence can be constructed recursively, and it is independent of $m$ ), and where the vectors $\mathbf{c}_{i}, i=2, \ldots, m$, are shifts of the vector $\mathbf{c}_{1}$ :

$$
\mathbf{c}_{2}=\left(0,0,1, \alpha_{1}, 0, \alpha_{3}, \ldots, 0, \alpha_{2 m-3}\right), \ldots \mathbf{c}_{m}=\left(0,0, \ldots, 1, \alpha_{1}\right) .
$$

For instance, the solutions space for $m=4$ is the $\mathbb{F}$-span of the columns of the following matrix:

$$
\left(\begin{array}{cccc}
1 & 0 & 0 & 0 \\
-\frac{1}{1-q} & 0 & 0 & 0 \\
\frac{q}{(1-q)^{2}\left(1-q^{3}\right)} & -\frac{1}{1-q} & 0 & 0 \\
0 & 0 & 1 & 0 \\
\frac{q^{2}\left(1+q^{2}\right)}{(1-q)^{3}\left(1-q^{3}\right)\left(1-q^{5}\right)} & \frac{q}{(1-q)^{2}\left(1-q^{3}\right)} & -\frac{1}{1-q} & \\
0 & 0 & 0 & 1 \\
\frac{q^{3}\left(q^{8}+q^{7}+3 q^{6}+2 q^{5}+3 q^{4}+2 q^{3}+3 q^{2}+q+1\right)}{(1-q)^{3}\left(1-q^{3}\right)^{2}\left(1-q^{5}\right)\left(1-q^{7}\right)} & \frac{q^{2}\left(1+q^{2}\right)}{(1-q)^{3}\left(1-q^{3}\right)\left(1-q^{5}\right)} & \frac{q}{(1-q)^{2}\left(1-q^{3}\right)} & -\frac{1}{1-q}
\end{array}\right)
$$

Since we already know that all solutions are $x$-invariant, we may work with the simpler matrix $\mathrm{M}^{\prime \prime \prime}(m, \infty)$ with $(i, j)$-entry

$$
\mathbf{e}(m, i, j, \infty)=\lim _{x \rightarrow \infty} \mathbf{e}(m, i, j, x)=\left(q^{2(m-i+1)-j} ; q\right)_{j}
$$

(where we assumed that $|q|<1$ in taking the limit) and consider

$$
\overline{\mathbf{a}}=(0,1,3,5, \ldots, 2 m-1) .
$$

Observe, that in each step of "working our way up" from the last equation corresponding to this particular sequence $\overline{\mathbf{a}}$ (as in the proof of Lemma 4.1), precisely one new variable has to be considered. I.e., starting with $\alpha_{1}=q-1$ from the last equation (see (34)), the next-to-last equation becomes a linear equation in a single variable, which, of course, can easily be solved. This explains the recursive construction of the solution vector $\mathbf{c}_{1}$.

Now observe that deleting the first two columns and the last row of $\mathrm{M}^{\prime \prime \prime}(m, \infty)$, and dividing all remaining rows by their first entry gives $\mathrm{M}^{\prime \prime \prime}(m-1, \infty)$, whence we may prepend two zeros to the solution $\mathbf{c}_{1}$ (as just described) for $m-1$ and obtain another solution of $\mathrm{M}^{\prime \prime \prime}(m, \infty)$. This makes clear that we find the $m$ solution vectors $\mathbf{c}_{1}, \ldots, \mathbf{c}_{m}$ (which obviously are linearly independent), as claimed above.

We already know that there exists an $x$-invariant solution of the equations corresponding to a general reversed admissible sequence $\overline{\mathbf{a}}$ (derived from some admissible sequence $\mathbf{a}$, as in the proof of Lemma 4.1), which may be viewed as a solution vector for (35) where all entries not in $\overline{\mathbf{a}}$ are set to zero. It is easy to see how to construct a solution vector with these "prescribed zeros" as a linear combination of $\mathbf{c}_{1}, \ldots, \mathbf{c}_{m}$.

\section{Acknowledgement}

I am grateful to Christian Krattenthaler for helpful discussions, and I am very grateful to the anonymous referees for their valuable comments, which greatly helped in improving the first version of this manuscript (of course, for all remaining flaws I accept full responsibility).

\section{References}

[1] M. Ciucu, T. Eisenkölbl, C. Krattenhaler, and D. Zare, Enumeration of lozenge tilings of hexagons with a central triangular hole, J. Combin. Theory Ser. A 95 (2001), 251-334.

[2] D. Condon, Lozenge Tiling function ratios for hexagons with dents on two sides, arXiv:2002.01988, February 2020. 
[3] I. M. Gessel and X. Viennot, Determinants, paths, and plane partitions, preprint, 1989.

[4] T. Lai, Ratio of tiling generating functions of semi-hexagons and quartered hexagons with dents, arXiv:2006.10900, June 2020.

[5] B. Lindström, On the vector representation of induced matroids, Bull. London Math. Soc. 5 (1973), 85-90.

[6] P. A. MacMahon, Combinatory Analysis, Volume 2, Cambridge University Press, 1916.

[7] M. P. Schützenberger, Une interprétation de certaines solutions de l'équation fonctionnelle: $f(x+y)=$ $f(x) f(y)$, C. R. Acad. Sci. Paris 236 (1953), 352-353. 